\title{
Checklist das Poaceae de áreas inundáveis e inundadas do nordeste do estado do Pará
}

\author{
Antônio Elielson Sousa da ROCHA ${ }^{1}$, Alba Lúcia Ferreira de Almeida LINS ${ }^{1}$
}

\section{RESUMO}

É apresentada uma relação de Poaceae coletadas em áreas inundáveis e inundadas do nordeste do Estado do Pará. São registrados 31 gêneros, compreendendo 83 espécies. Os grupos mais bem representados são os gêneros Paspalum e Panicum (17 e 18 taxa, respectivamente) e a tribo Paniceae (57 taxa). São fornecidos posição sistemática, comentários sobre afinidades taxonômicas, distribuição geográfica, ciclo de vida, coleçôes botânicas e ilustração da espigueta das espécies.

PALAVRAS-CHAVE: Poaceae, Amazônia, áreas úmidas

\section{Checklist of Poaceae in periodically and permanently flooded areas in northeastern region of Pará state}

\section{ABSTRACT}

A list of Poaceae collected in periodically flooded and permanently flooded areas in the paraense northeastern region of Pará State is presented. Currently, 31 genera and 83 species are recognized. The most representative groups are Paspalum, Panicum (17 and 18 taxa, respectively), and the tribe Paniceae (57 taxa). The systematic position, taxonomic affinities, geographical distribution, vegetative cicle, a list of examined specimens, and illustrations of spikelet of each species are given.

KEYWORDS: Poaceae, Amazônia, wetlands 


\section{INTRODUÇÃO}

A região amazônica é portadora de imensas áreas úmidas, sazonal ou permanentemente inundadas, onde se desenvolvem desde plantas estritamente aquáticas a anfíbias, apresentando adaptações que possibilitam a rápida colonização em diferentes tipos de ambientes. Nos ambientes abertos as Poaceae estão entre as dominantes, algumas espécies terrestres, bem adaptadas à fase aquática e outras consideradas aquáticas são muito bem adaptadas à fase terrestre (Junk \& Piedade, 1993; Conserva \& Piedade, 1998).

No nordeste paraense as formaçóes campestres aparecem como interrupçôes da floresta. Podendo ser destacados principalmente as savanas com trechos mal drenados, os pirizais, bolsóes marginais aos rios, os campos de várzea, os campos litorâneos e os campos brejosos de restinga (Pires, 1973).

O conhecimento que se tem sobre as espécies de Poaceae que compóe estes ambientes é limitado. Apesar da forte ação antrópica sofrida, muitos destes campos, nunca foram explorados botanicamente, quando isso acontece, apenas listagens são apresentadas.

Em virtude da boa representatividade da família nestes ambientes e por ser um taxon de reconhecido interesse econômico, este estudo dará ênfase à diversidade das Poaceae em áreas úmidas ao longo do nordeste paraense.

Através de um checklist comentado e ilustrado das espécies, busca-se ampliar o conhecimento sobre as Poaceae na região, além de se criar um indicativo importante aos estudos sobre tolerância de capins tropicais ao alagamento, fator limitante para atividade pecuária na regiáo.

\section{MATERIAL E MÉTODOS}

A lista apresentada foi elaborada a partir de coletas dos autores realizadas em áreas úmidas, ao longo do nordeste paraense, especialmente nos municípios de Belém, Barcarena, Vigia, Marapanim, Maracanã, Santarém-Novo, Salinópolis e Bragança; além de todo material depositado nos herbários do Museu Paraense Emílio Goeldi (MG) e da EMBRAPA, Amazônia Oriental (IAN) (Holmgren \& Holmgren, 1998).

Espécies exóticas ou em escape de cultivo foram incluídas.

Espécies dos gêneros Pariana e Olyra (Bambusoideae: Olyreae), tipicamente de floresta; por terem sido coletadas, pelo autor, em ambiente alagado foram incluídas neste estudo.

As amostras coletadas foram processadas, seguindo-se a metodologia descrita por Filgueiras (1992) e depositadas no Herbário João Murça Pires (MG).
As identificaçôes taxonômicas foram efetuadas através da análise morfológica dos caracteres florais e vegetativos; auxílio de diagnoses, descriçôes e chaves analíticas encontradas na bibliografia especializada (Black, 1950; Renvoize, 1984; Judziewicz et al., 1990; Longhi-Wagner et al., 2001, entre outros). Como também tipos e fotos de tipos, quando disponíveis.

A classificação em subfamília seguiu GPWG (2001) exceto para o gênero Isachne, incluído em Micrairoideae, segundo Sánchez-Ken et al. (2007). A terminologia empregada está de acordo com Longhi-Wagner et al. (2001).

A distribuição geográfica das espécies foi obtida através da literatura, especialmente Judziewicz et al. (1990); LonghiWagner et al. (2001) Rodrigues-da-Silva \& Filgueiras (2003).

Todas as escalas das figuras correspondem a um milímetro.

\section{RESULTADOS E DISCUSSÃO}

Nas áreas amostradas no nordeste paraense foram encontradas seis subfamílias, oito tribos, 31 gêneros, compreendendo 83 espécies de Poaceae. Deste total, 71 espécies são nativas e 12 exóticas (Tabela 1).

Os gêneros mais representativos foram: Paspalum com 17 e Panicum com 18 espécies; a tribo Paniceae (Panicoideae) com 57 espécies foi a mais representada.

Da totalidade das espécies, 38 apresentam padrão de distribuição amplo interamericano, compreendendo as Américas do Norte, Central e Sul. Entre as espécies que apresentam este padrão fitogeográfico, Eragrostis hypnoides, Panicum dichotomiflorum e Spartina alterniflora são as que apresentam a distribuição mais ampla, a última sempre associada ao ambiente de restinga, podendo ser encontrada totalmente submersa nos baixios da região entre-marés.

Onze espécies são restritas da América do Sul, com Paspalum conspersum e $P$. intermedium, amplamente distribuídas e as demais, restritas a Amazônia e paises adjacentes, sendo Panicum pseudisachne endêmica do Brasil.

Quinze espécies ocorrem na América do Sul, com algumas se estendendo até o Panamá, Nicarágua ou Cuba; entre elas, Reimarochloa acuta, que possui distribuição mais ampla, sempre associada aos campos de solo argiloso.

Dezoito espécies estendem suas distribuiçóes além das fronteiras do continente, sendo 8 cosmopolitas (6 delas exóticas) e 10 pantropicais (6 também exóticas). (Tabela 1). Das 10 pantropicais, apenas Sporobolus virginicus ocorre caracteristicamente em ambiente de restinga. No nordeste do estado, esta espécie pode ser encontrada nos campos 
Tabela 1 - Lista de espécies de Poaceae coletadas em áreas úmidas no nordeste paraense. Ciclo de vida: $A=$ anual; $P=$ perene. $\left({ }^{\star}\right)$ Espécie exótica.

\begin{tabular}{|c|c|c|c|}
\hline Espécie & $\begin{array}{l}\text { Ciclo de } \\
\text { vida }\end{array}$ & Distribuição geográfica & Material testemunho \\
\hline \multicolumn{4}{|l|}{ BAMBUSOIDEAE } \\
\hline \multicolumn{4}{|l|}{ OLYREAE } \\
\hline Olyra ecaudata Doell & $\mathrm{P}$ & $\begin{array}{l}\text { América do Sul: Mesoámerica, norte e oeste da } \\
\text { América do Sul e Brasil. }\end{array}$ & D. Daly 1937 - MG \\
\hline 0. Iongifolia Kunth & $P$ & Norte e oeste da América do Sul e Brasil. & Costa-Neto 228 - MG \\
\hline Pariana campestris Aubl. & $\mathrm{P}$ & Norte e oeste da América do Sul e Brasil. & A.E.S. Rocha 325 - MG \\
\hline \multicolumn{4}{|l|}{ EHRHARTOIDEAE } \\
\hline \multicolumn{4}{|l|}{ ORYZEAE } \\
\hline Leersia hexandra Sw. & $\mathrm{P}$ & Cosmopolita. & A.E.S. Rocha 601 - MG \\
\hline Luziola bahiensis (Steud.) Hitchc. & $\mathrm{P}$ & Dos EUA até a Argentina. & J.M. Pires 205 - IAN \\
\hline L. spruceana Benth. ex Döll & $P$ & México, Venezuela, Brasil e Paraguai. & A.E.S. Rocha 606 - MG \\
\hline Oryza grandiglumis (Doell) Prod. & $\mathrm{P}$ & Norte e oeste da América do Sul e Brasil. & A. Silva 295 - MG \\
\hline 0. Iatifolia Desv. & $\mathrm{P}$ & Do México ao Brasil. & S. Almeida $332-$ MG \\
\hline 0. rufipogon Griff. & $P$ & Pantropical. * & G.A. Black 8576 - MG \\
\hline O. sativa L. & A & Pantropical. * (em escape de cultura) & P. Cavalcante 967 - MG \\
\hline \multicolumn{4}{|l|}{ ARUNDINOIDEAE } \\
\hline \multicolumn{4}{|l|}{ ARUNDINEAE } \\
\hline Phragmites australis (Cav.) Trin ex Steud. & $\mathrm{P}$ & Cosmopolita. & G.A. Black 50-9019 - IAN \\
\hline \multicolumn{4}{|l|}{ CHLORIDOIDEAE } \\
\hline \multicolumn{4}{|l|}{ ERAGROSTIDEAE } \\
\hline Eragrostis acutiflora (Kunth) Nees & $\mathrm{P}$ & Do México ao Brasil. & W.A. Egler 1037 - MG \\
\hline E. hypnoides (Lam.) Britton & A & Do Canadá à Argentina. & A.E.S. Rocha 614 - MG \\
\hline E. glomerata (Walter)L.H.Dewey & A & Dos EUA até a Argentina. & G.A. Black 9188 - MG \\
\hline E. pilosa (L.) P. Beauv. & A & Cosmopolita. * & $\begin{array}{l}\text { J.M. Pires \& G.A. Black } 383- \\
\text { IAN }\end{array}$ \\
\hline Leptochloa scabra Nees & A & Dos EUA ao Paraguai. & A. Serrão 16 - IAN \\
\hline Sporobolus virginicus (L.) Kunth. & $\mathrm{P}$ & Pantropical & A.E.S. Rocha 14 - MG \\
\hline \multicolumn{4}{|l|}{ CYNODONTEAE } \\
\hline Cynodon dactylon (L.) Pers. & $\mathrm{P}$ & Cosmopolita. * & N. Rosa 5556a - MG \\
\hline Spartina alterniflora Loisel. & $P$ & Canadá à Argentina. & M.N. Bastos 1915 - MG \\
\hline \multicolumn{4}{|l|}{ PANICOIDEAE } \\
\hline \multicolumn{4}{|l|}{ PANICEAE } \\
\hline Acroceras zizanioides (Kunth) Dandy & $\mathrm{P}$ & Pantropical. & A.E.S. Rocha 194 - MG \\
\hline Axonopus purpusii (Mez) Chase & $\mathrm{P}$ & México ao Brasil. & P. Cavalcante 196 - MG \\
\hline $\begin{array}{l}\text { Cyphonanthus discrepans (Döll) Zuloaga \& } \\
\text { Morrone }\end{array}$ & $P$ & Cuba, Venezuela, Guianas e Brasil. & A.E.S. Rocha 01 \\
\hline Echinochloa colona (L.) Link. & A & Cosmopolita. * & A.E.S. Rocha 196 - MG \\
\hline E. crusgallis (L.) P. Beauv. & $A$ & Cosmopolita. ${ }^{*}$ & V. Miranda 3241 - MG \\
\hline E. crus-pavonis (Kunth) Schult. & $A$ & Cosmopolita. * & G. Davidse 18088 - MG \\
\hline E. polystachya (Kunth) Hitchc. & $\mathrm{P}$ & México à Argentina. & J. Huber 277 - MG \\
\hline Eriochloa distachya Kunth & A & Panamá, Bolívia, Brasil, Colômbia, Peru e Venezuela. & M. Guedes 2397 - MG \\
\hline E. punctata (L.) Desv. ex W. Ham. & $\mathrm{P}$ & Dos EUA à Argentina. & E. Oliveira 5012 - IAN \\
\hline Hymenachne amplexicaulis (Rudge) Nees & $\mathrm{P}$ & México à Argentina. & A.E.S. Rocha $207-M G$ \\
\hline H. donacifolia (Raddi) Chase & $\mathrm{P}$ & Costa Rica, Cuba, Bolívia, Argentina, Guianas e Brasil. & Davidse 18114 - MG \\
\hline Panicum aquaticum Poir. & $P$ & México à Argentina. & J. Swallen 4915 - MG \\
\hline P. caricoides Nees ex Trin. & $\mathrm{P}$ & México ao Brasil. & N.T. Silva 156 - IAN \\
\hline P. cyanescens Nees ex Trin. & $\mathrm{P}$ & México ao Brasil. & A.E.S. Rocha 122 - MG \\
\hline P. dichotomiflorum Michx. & A & Canadá à Argentina. & M. Guedes 2415 - MG \\
\hline P. elephantipes Nees ex Trin. & $\mathrm{P}$ & México à Argentina. & G.A. Black 9188 - MG \\
\hline
\end{tabular}




\begin{tabular}{|c|c|c|c|}
\hline Espécie & $\begin{array}{l}\text { Ciclo de } \\
\text { vida }\end{array}$ & Distribuição geográfica & Material testemunho \\
\hline P. grande Hitchc. \& Chase & $\mathrm{P}$ & $\begin{array}{l}\text { Cuba, Guatemala, Venezuela, Trindade, Guianas, Peru } \\
\text { e Brasil. }\end{array}$ & J.M. Pires 4858 - IAN \\
\hline P. hylaeicum Mez & $P$ & México à Argentina. & R.L. Fróes 27842- IAN \\
\hline P. mertensii Roth & $P$ & México à Argentina. & A.E.S. Rocha 610 - MG \\
\hline P. micranthum Kunth & A & Venezuela, Guianas e Brasil. & R.L. Fróes 27842 - MG \\
\hline P. nervosum Lam. & $\mathrm{P}$ & Venezuela, Trindade e Brasil. & N. Rosa $952-M G$ \\
\hline P. parvifolium Lam. & $\mathrm{P}$ & México a Argentina. & J. Huber 2686 - MG \\
\hline P. pilosum Sw. & $P$ & Antilhas, México à Argentina. & A. Lins $361-M G$ \\
\hline P. polygonatum Schrad. & $\mathrm{P}$ & México à Argentina. & V. Miranda 7048 - MG \\
\hline P. pseudisachne Mez & $\mathrm{P}$ & Brasil. & L. Swallem 5037 - MG \\
\hline P. pyrularium Hitchc. \& Chase & A & Panamá, Colômbia, Venezuela, Guianas e Brasil. & J. Swallem 4936 - MG \\
\hline P. repens $\mathrm{L}$. & $\mathrm{P}$ & Cosmopolita. * & Miranda 3238 - MG \\
\hline P. stenodes Griseb. & $\mathrm{P}$ & Costa Rica ao Brasil. & M. Silva 4966 - MG \\
\hline P. stoloniferum Poir. & $P$ & México à Argentina. & J. M. Pires $602-$ MG \\
\hline Paratheria prostrata Griseb. & $\mathrm{P}$ & Pantropical. ${ }^{*}$ & M.N.C. Bastos 1216 - MG \\
\hline Paspalidium geminatum (Forssk.) Stapf. & $P$ & Pantropical. * & A.E.S. Rocha 195 - MG \\
\hline Paspalum conjugatum Berg. & $\mathrm{P}$ & Pantropical. & E. Oliveira 3456 - IAN \\
\hline P. conspersum Schrad. & $\mathrm{P}$ & América do Sul. & R. Lima $26-M G$ \\
\hline P. coryphaeum Trin. & $P$ & Panamá, Trindade, Guianas e Brasil. & V. Miranda 3116 - MG \\
\hline P. densum Poir. & $P$ & América Central e Sul. & V. Miranda 3135 - MG \\
\hline P. denticulatum Trin. & $\mathrm{P}$ & Dos EUA à Argentina. & M. Guedes 2337 - MG \\
\hline P. fasciculatum Willd. ex Flüggé & $P$ & México à Argentina. & J. Swallen 3165 - MG \\
\hline P. intermedium Munro ex Monong \& Britton & $\mathrm{P}$ & Brasil, Paraguai, Uruguai e Argentina. & J. Huber 497 - MG \\
\hline P. melanospermum Desv. ex Poir. & A & Panamá, Colômbia, Venezuela, Guianas e Brasil. & G. Davidse 18137 - MG \\
\hline P. orbiculatum Poir. & A & México, Cuba ao Paraguai. & A.E.S. Rocha 197 - MG \\
\hline P. pleostachyum Doell & $\mathrm{P}$ & Republica Dominicana, Haiti, Porto Rico e Brasil. & F. Lima $10768-M G$ \\
\hline P. plicatulum Michx. & $\mathrm{P}$ & Dos EUA à Argentina. & J. Elias 225 - MG \\
\hline P. pulchellum Kunth & $\mathrm{P}$ & México ao Brasil. & E. Oliveira 3224 - IAN \\
\hline P. repens Berg. & $\mathrm{P}$ & Dos EUA ao Brasil. & A.E.S. Rocha 245 - MG \\
\hline P. trichophyllum Henrard. & A & Bolívia e Brasil. & G.A. Black 52-14387 - IAN \\
\hline P. vaginatum $\mathrm{Sw}$. & $\mathrm{P}$ & Pantropical. & A.E.S. Rocha $04-M G$ \\
\hline P. virgatum $\mathrm{L}$. & $\mathrm{P}$ & México à Argentina. & L. Carreira 1937 - MG \\
\hline P. wrightii Hitchc. \& Chase & $\mathrm{P}$ & Cuba, Venezuela, Guianas e Brasil. & J.M. Pires 4476 - IAN \\
\hline Reimarochloa aberrans (Doell) Chase & $\mathrm{P}$ & Guiana Francesa e Brasil. & A.E.S. Rocha $02-M G$ \\
\hline R. acuta (Flüggé) Hitchc. & $P$ & $\begin{array}{c}\text { El Salvador, Honduras, Cuba, Bolivia, Guianas, Brasil, } \\
\text { Paraguai e Argentina. }\end{array}$ & Costa-Neto 271 - MG \\
\hline $\begin{array}{l}\text { Sacciolepis angustissima } \\
\text { (Hochst. ex Steud.) Kuhlm. }\end{array}$ & A & Colômbia, Venezuela, Bolívia, Guianas e Brasil. & V. Miranda $3110-M G$ \\
\hline S. myuros (Lam.) Chase & A & México ao Brasil. & M.P. Neves 240 - MG \\
\hline S. striata (L.) Nash & $\mathrm{P}$ & Dos EUA ao Brasil. & A.E.S. Rocha 604 - MG \\
\hline S. vilvoides (Trin.) Chase & A & Cuba, Venezuela, Guianas, Paraguai e Brasil. & A.E.S. Rocha 13 - MG \\
\hline Setaria parviflora (Poir.) Kerguélen & $\mathrm{P}$ & Dos EUA à Argentina. & W. Egler 1526 - MG \\
\hline Steinchisma laxa (Sw.) Zuloaga & $\mathrm{P}$ & México à Argentina. & A.E.S. Rocha 607 - MG \\
\hline Urochloa mutica (Forssk) T.Q. Nguyen & $\mathrm{P}$ & Pantropical.* & N. Menezes s.n. - IAN \\
\hline \multicolumn{4}{|l|}{ ANDROPOGONEAE } \\
\hline Andropogon bicornis L. & $\mathrm{P}$ & México à Argentina. & V. Miranda 3240 - MG \\
\hline A. selloanus (Hack.) Hack. & $\mathrm{P}$ & México à Argentina. & W. Egler 1281 - MG \\
\hline A. virgatus Desv. ex Ham. & $P$ & México à Argentina. & M. Silva 4968 - MG \\
\hline Eriochrysis cayennensis P. Beauv. & $P$ & México à Argentina. & P. Cavalcante 907 - MG \\
\hline Hemarthria altissima (Poir.) Stapf \& Hubb. & $\mathrm{P}$ & Pantropical $^{*}$ & M. Guedes 2389 - MG \\
\hline
\end{tabular}


Tabela 1 - Continuação

\begin{tabular}{lccc}
\hline Espécie & $\begin{array}{c}\text { Ciclo de } \\
\text { vida }\end{array}$ & Distribuição geográfica & Material testemunho \\
\hline Ischaemum guianense Kunth ex Hack. & $\mathrm{P}$ & Venezuela, Guianas e Brasil. & A. Lins 129 - MG \\
MICRAIROIDEAE & & & \\
ISACHNEAE & $\mathrm{P}$ & México ao Brasil. & V. Miranda 21039 - MG \\
\hline Isachne polygonoides (Lam.) Doell & P.
\end{tabular}

inundáveis hipersalinos, denominados "apicuns", onde praticamente só ela se estabelece.

Sessenta e cinco espécies apresentam ciclo perene e dezoito anuais (Tabela 1). Existem razôes para se esperar que espécies de ciclo perene predominem. As "canaranas", por exemplo, (Echinocloa polystachya, Paspalum conspersum, P. repens, P. fasciculatum, Hymenachne amplexicaule e Luziola spruceana) que geram alta densidade populacional através da reprodução vegetativa, levam vantagem neste tipo de ambiente, onde o estabelecimento de plântulas é impedido pela sedimentação ou condiçôes anóxicas do solo (Worbes, 1997).

Eragrostis glomerata e E. hypnoides são espécies do gênero mais adaptadas às áreas palustres, sendo encontradas em áreas inundadas do pantanal por período prolongado (Allem \& Valls, 1987).

Das espécies de Panicum levantadas: P. aquaticum, $P$. dichotomiflorum, P. repens e P. elephantipes pertencem ao subg. Panicum, seçáo Dichotomiflora, sendo bastante semelhantes entre si, diferindo basicamente por serem: $P$. aquaticum, $P$. elephantipes e $P$. repens perenes e $P$. dichotomiflorum anual. A diferença entre $P$. aquaticum, $P$. elephantipes e $P$. repens está no comprimento da espigueta: de 4,5-5,5 em $P$. elephantipes e 2,5-3,5 $\mathrm{mm}$ em $P$. aquaticum e $P$. repens, sendo que em $P$. aquaticum o ápice da gluma inferior é agudo ou acuminado e em $P$. repens é truncado. Todas são invasoras de cultivo e jardim (Guglieri et al., 2004; Guglieri et al., 2007).

Panicum parvifolium, P. micranthum, P. cyanescens, $P$. pyrularium, P. pseudisachne e $P$. nervosum pertencem ao subg. Phanopyrum seção Parvifolia, compartilham de um tipo similar de inflorescência, uma panícula laxa e pauciflora, com espiguetas geralmente de pedicelos longos (Aliscioni $e t$ al., 2003).

Panicum pseudisachne e P. pyrularium apresentam espiguetas pilosas, diferindo pela base da lâmina: amplexicaule em $P$. pyrularium e arredondada a subcordada em $P$. pseudisachne. As demais espécies apresentam espiguetas glabras, com $P$. parvifolia apresentando inflorescência e lâmina menores (1-5 cm compr. e 1,5-3 cm compr.) e $P$. nervosum, maiores (10-20 $\mathrm{cm}$ compr. e 11-18 cm compr.). P. cyanescens e P. micranthum apresentam porte intermediário $(5-14 \mathrm{~cm}$ compr. e $4-11 \mathrm{~cm}$ compr.), porém diferem na ráquis da inflorescência: escabrosa em $P$. cyanescens e lisa em P. micranthum.
Panicum hylaeicum, P. pilosum e P. polygonatum pertencem ao subg. Phanopyrum, seção laxa. Assemelham-se no porte, inflorescência e espigueta; diferem pelas seguintes caracterísitcas: $P$. pilosum apresenta ausência de lígula; $P$. polygonatum apresenta pseudo-pecíolo e P. hylaeicum possui base da folha cordada.

Panicum caricoides e P. stenodes pertencem ao subgênero Agrostoides seção Tenera. Vegetativamente são muito semelhantes. Diferenciam-se pelas dimensóes da espigueta e entrenó entre as glumas, maiores em $P$. caricoides.

Dentre as espécies de Paspalum levantadas Paspalum melanospermum, $P$. plicatulum e $P$. wrightii pertencem ao subgênero Paspalum, grupo Plicatula. Assemelham-se pelo lema do antécio inferior plicado transversalmente. $P$. melanospermum se separa das demais por ser anual; $P$. plicatulum e $P$. wrightii são perenes e apresentam espiguetas aos pares, porém diferem pelos ramos da inflorescência, os basais iguais aos apicais em $P$. plicatulum e os basais maiores que os apicais em $P$. wrightii.

Paspalum distichum (não levantado neste estudo) e $P$. vaginatum pertencem a seção Disticha. Assemelham-se pela disposição das folhas dísticas e ramos unilaterais conjugados, e diferem pela gluma superior: pubérula em $P$. distichum e glabra em $P$. vaginatum.

Espécies do gênero Paspalum grupo Dissecta são todas aquáticas ou subaquáticas. Morrone et al. (1996) cita duas espécies deste grupo para o Estado do Pará: P. morichalense Davidse, Zuloaga \& Filg. e $P$. repens; porém, no presente estudo, foi levantada amostra apenas da segunda.

Paspalum repens se distingue das demais espécies do grupo por apresentar maior número de ramos na inflorescência, entre 20-30 ramos, com apenas uma fileira de espigueta por ramo. Seguindo conceito de Morrone et al. (1996), Paspalum pyramidale Nees e P. fluitans (Elliott) Kunth serão considerados sinônimos de $P$. repens.

As espécies do gênero Echinochloa habitam preferencialmente ambientes úmidos e alagados. $\mathrm{Na}$ análise dos espécimes coletados e herborizados foram levantadas quatro espécies do gênero, a separação entre elas foi feita, basicamente, através de caracteres da espigueta.

Hymenachne amplexicaule e $H$. donacifolia podem ser reconhecidas, especialmente, pelos ramos da inflorescência: 
adpressos em $H$. amplexicaule e ascendentes em $H$. donacifolia.

Baseado na análise das coleções de Luziola depositadas nos herbários visitados, inclusive uma coleção de Spruce de 1848 (possivelmente isótipo), náo foi possível fazer a separação de material identificado como Luziola subintegra de L. spruceana, desta forma, todo material foi tratado como sendo L. spruceana.

Das espécies de Oryza citadas por Black (1950) para a Amazônia, estamos adotando o conceito de Judziewicz et al. (1990) que consideram $O$. alta sob O. latifolia; $O$. glumaepatula e $O$. perennis sob $O$. rufipogon.

Com exceção de $O$. sativa, que não apresenta arista, as demais se separam, basicamente, pelo comprimento das glumas.
Algumas amostras de Acroceras zizanioides encontravamse determinadas, erroneamente, como Homolepis isocalycia (G.Mey.) Chase. Estes táxons são bastante semelhantes vegetativamente, diferindo principalmente no tamanho da gluma inferior, que em $H$. isocalycia é do comprimento da espigueta e com 5-9 nervuras; enquanto em $A$. zizanioides alcança três quartos do comprimento da espigueta, sendo 3 nervada.

As espécies Cynodon dactylon, Paspalum vaginatum e Paspalum pleostachyum, assim como Spartina alterniflora e Sporobolus virginicus, citadas anteriormente, ocorrem preferencialmente na regiấo litorânea, sendo encontradas em áreas de restinga e manguezal no estado do Pará.

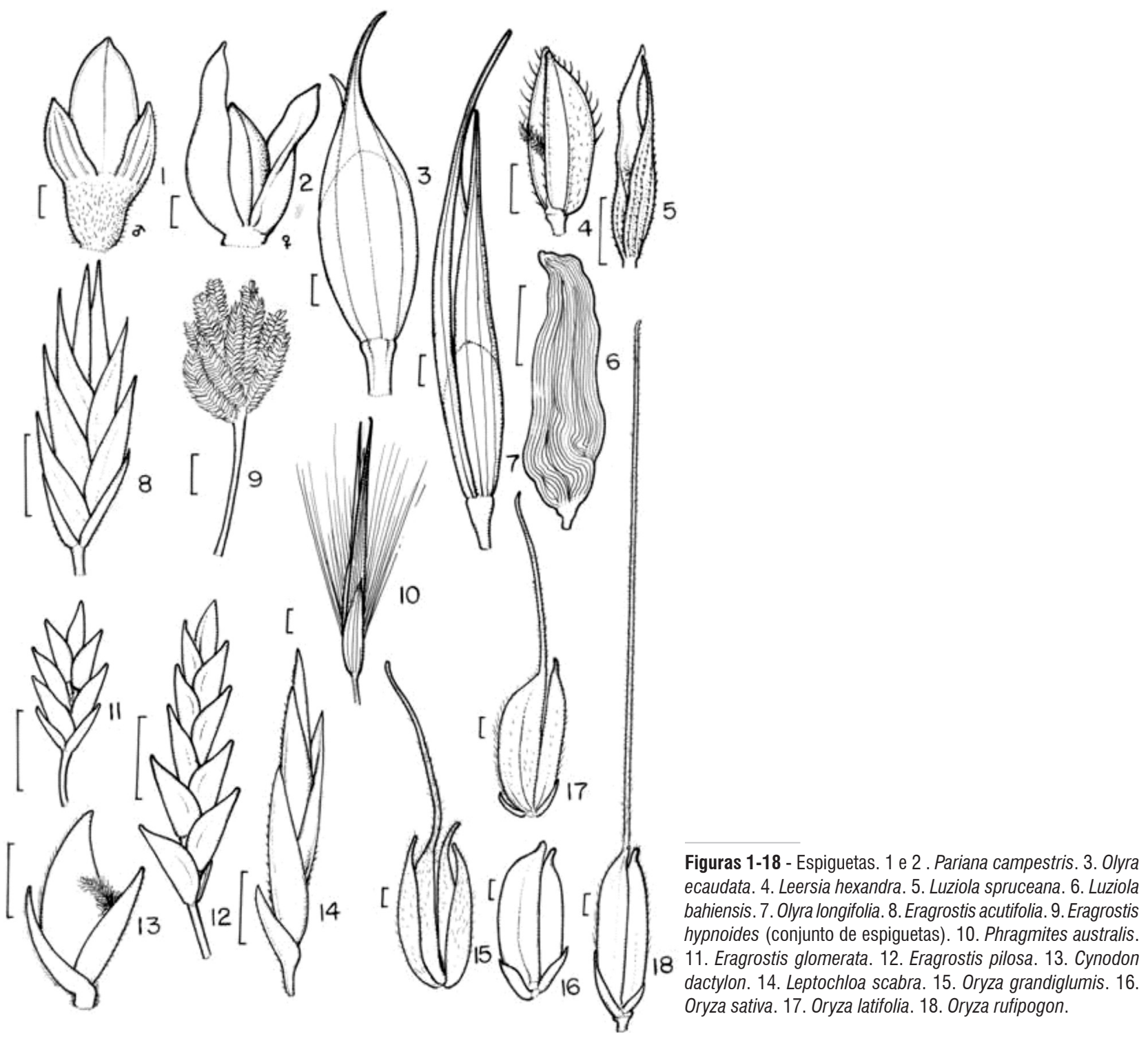




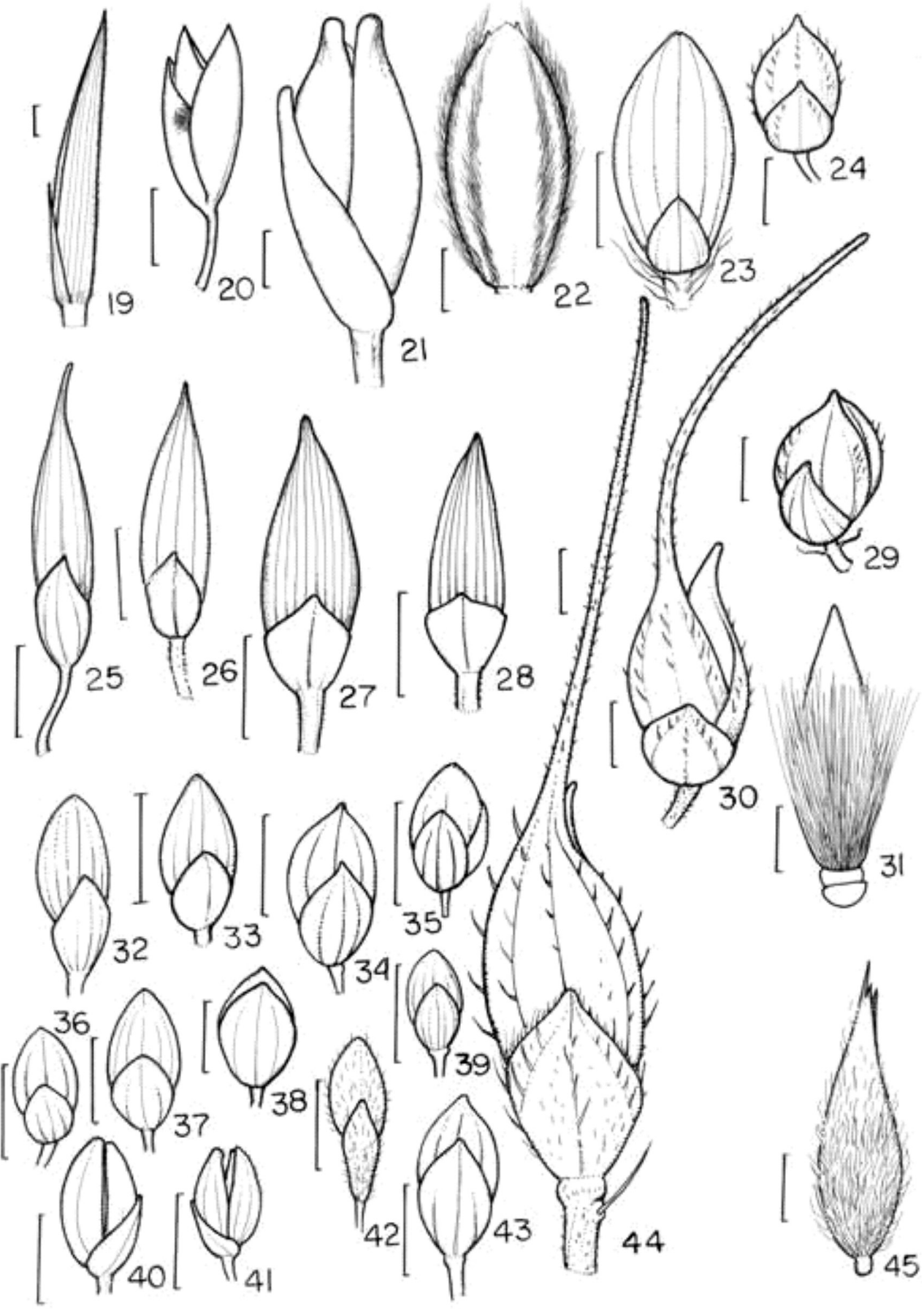

Figuras 19-45 - Espiguetas. 19. Spartina alterniflora. 20. Sporobolus virginicus. 21. Acroceras zizanioides. 22. Axonopus purpusii. 23. Urochloa mutica. 24. Echinochloa colona. 25. Hymenachne amplexicaulis. 26. Hymenachne donacifolia. 27. Panicum aquaticum. 28. Panicum dichotomiflorum. 29. Echinochloa crus-pavonis. 30. Echinochloa crus-gallis. 31. Eriochloa distachya. 32. Panicum caricoides. 33. Panicum stenodes. 34. Panicum cyanescens. 35. Panicum parvifolium. 36. Panicum hylaeicum. 37. Panicum pilosum. 38. Panicum pseudisachne. 39. Panicum micranthum. 40. Steinchisma laxa. 41. Panicum polygonatum. 42. Panicum pyrularium. 43 Panicum nervosum. 44. Echinochloa polystachya. 45. Eriochloa punctata. 


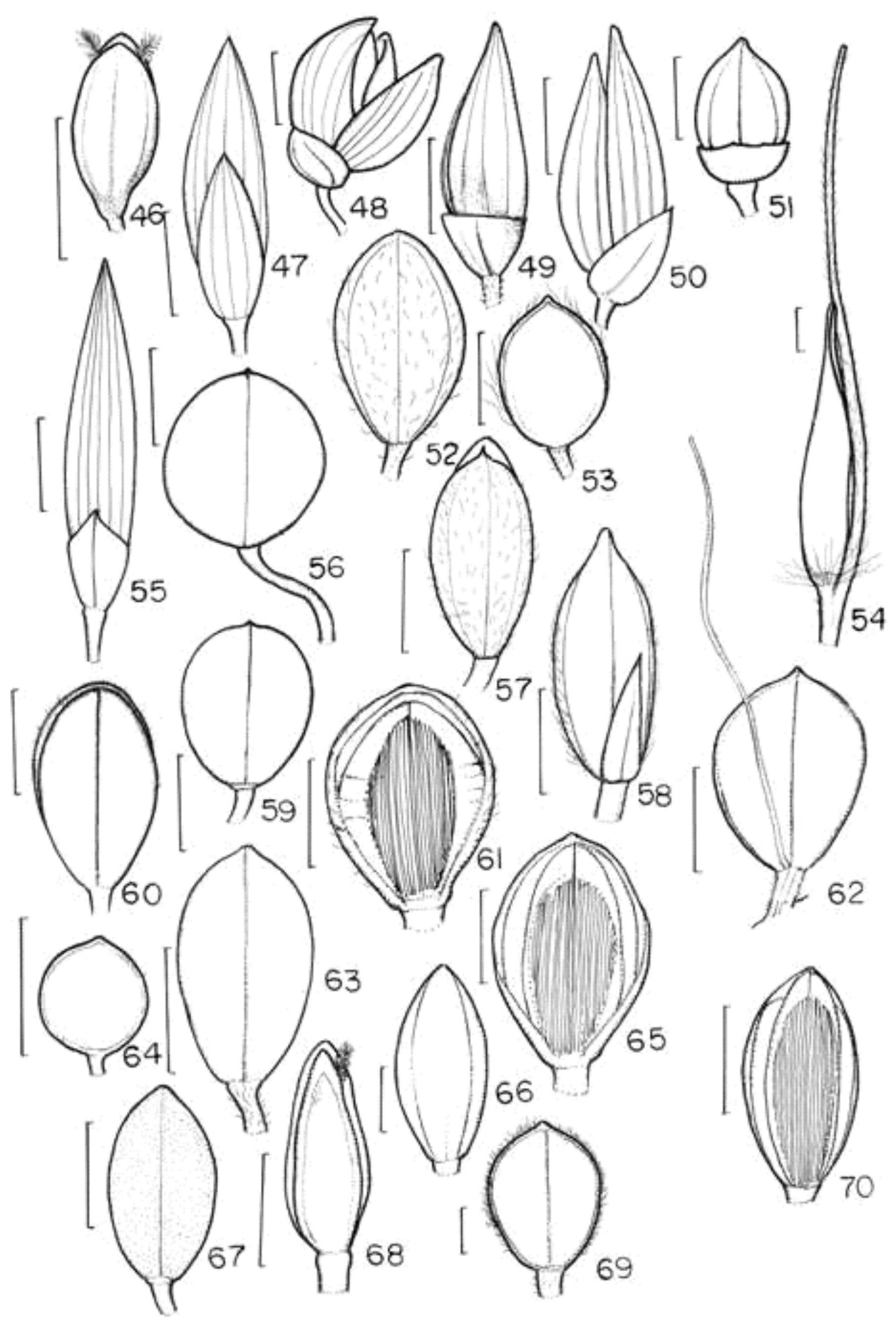

Figuras 46-70 - Espiguetas. 46. Cyphonanthus discrepans. 47. Panicum grande. 48. Panicum mertensii. 49. Panicum repens. 50. Panicum stoloniferum. 51. Paspalidium geminatum. 52. Paspalum conspersum. 53. Paspalum conjugatum. 54. Paratheria prostrata. 55. Panicum elephantipes. 56. Paspalum densum. 57. Paspalum coryphaeum. 58. Paspalum fasciculatum. 59. Paspalum intermedium. 60. Paspalum trichophyllum. 61. Paspalum melanospermum. 62. Paspalum denticulatum. 63. Paspalum pleostachyum. 64. Paspalum orbiculatum. 65. Paspalum plicatulum. 66. Paspalum vaginatum. 67. Paspalum pulchellum. 68. Paspalum repens. 69. Paspalum virgatum. 70. Paspalum wrightii. 


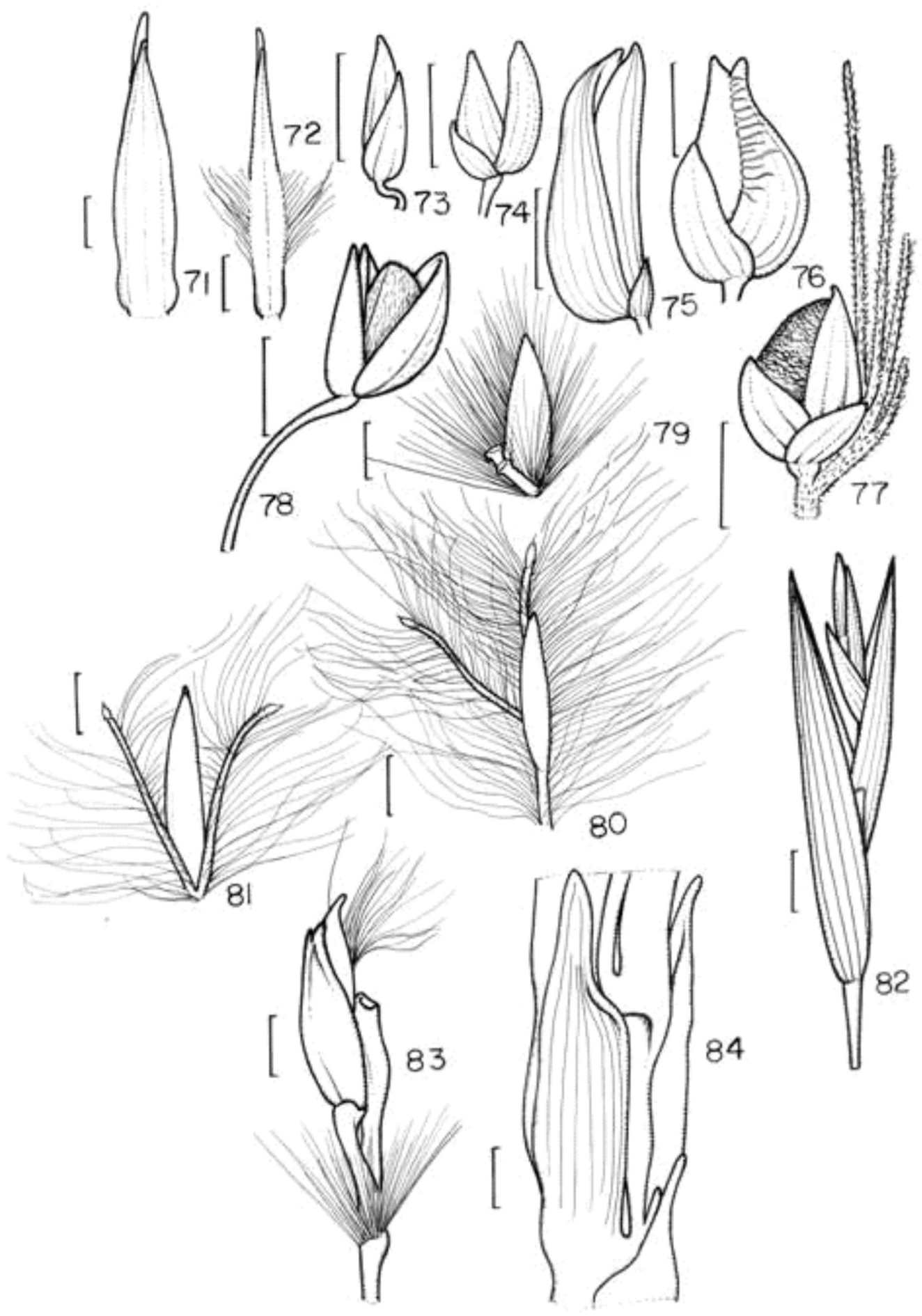

Figuras 71-84 - Espiguetas. 71. Reimarochloa aberrans. 72. Reimarochloa acuta. 73. Sacciolepis angustissima. 74. Sacciolepis myurus. 75. Sacciolepis striata. 76. Sacciolepis vilvoides. 77. Setaria parviflora. 78. Isachne polygonoides. 79. Eriochrysis cayennensis. 80. Andropogon bicornis. 81. Andropogon selloanus. 82. Andropogon virgatus (conjunto de espiguetas). 83. Ischaemum guianense. 84. Hemarthria altissima. 


\section{AGRADECIMENTOS}

Os autores agradecem a Helena Josiane Souza, funcionário do herbário IAN, a Dra. Ana Luiza Ilkiu-Borges pela revisão do abstract e aos referees pelas valiosas sugestôes.

\section{BIBLIOGRAFIA CITADA}

Allem, A.C.; Valls, J.F.M. 1987. Recursos forrageiros nativos do Pantanal Mato-Grossense. EMBRAPA, Brasília. 339p.

Aliscioni, S.S.; Giussani, L.M.; Zuloaga, F.O.; Kellogg, E.A. 2003. A molecular phylogeny of Panicum (Poaceae: Paniceae): tests of monophyly and phylogenetic placement within the Panicoideae. American Journal of Botany, 90:796-821.

Black, G. A. 1950. Os capins aquáticos da Amazônia, Belém. Bol. Técn. Inst. Agron. N., 19: 53-94.

Conserva, A.S.; Piedade, M.T.F. 1998. Influence of floodpulse and land-use on the composition of herbaceus species on a floodplain in central Amazonia. Verh.Internat. Verein. Limnol, 26: 994-995.

Filgueiras, T.S. 1992. Coleta, montagem e preservação de Gramíneas para estudos científicos. Boletim Informativo da UNB/Herbário, 2: $18-25$.

GPWG [Grass Phylogeny Working Group]. 2001. Phylogeny and subfamilial classification of the grasses (Poaceae). Annals of the Missouri Botanical Garden, 88: 373-457.

Guglieri, A.; Zuloaga, F.O.; Longhi-Wagner, H.M. 2004. Sinopse das espécies de Panicum L. subg. Panicum (Poaceae, Paniceae) ocorrentes no Brasil. Acta Bot. Bras., 18: 359-367.

Guglieri, A; Longhi-Wagner, H.M.; Zuloaga, F.O. 2007. Panicum sect. Dichotomiflora (Hitchc. \& Chase) Honda e P. sect. Virgata Hitchc. \& Chase ex Pilg. (Poaceae: Panicoideae: Paniceae) no Brasil. Acta Bot. Bras., 21(4): 785-805.

Holmgren, P. K., and N. H. Holmgren. 1998 [continuously updated]. Index Herbariorum: A global directory of public herbaria and associated staff. New York Botanical Garden's Virtual Herbarium. http://sweetgum.nybg.org/ih/
Judziewicz, E.J. 1990. Family 187. Poaceae (Gramineae). In: Flora of the Guianas. Series A: Phanerogams. v.8. (A.R.A. Gorts-van Rijn). Koeltz Scientific Books, Königstein, p:1-722.

Junk,W.; Piedade, M.T.F. 1993. Herbaceus plants of the amazon floodplain near Manaus: species diversity and adaptations to the flood pulse. Amazoniana, 12(3/4):467-484.

Longhi-Wagner, H. M., Bittrich, V., Wanderley, M. G. L. \& Shepherd, G. J. (Eds.) 2001. Poaceae. In: Wanderley, M. G. L., Shepherd, G. J. \& Giulietti, A. M. (Coords.) Flora Fanerogâmica do Estado de São Paulo. São Paulo: Hucitec. 292 p.

Morrone, O; Vega, A.S.; Zuloaga, F.O. 1996. Revisión de las especies del género Paspalum L. (Poaceae: Panicoideae: Paniceae), grupo Dissecta (s. str.). Candollea, 51(1): 103-138.

Pires, J.M. 1973. Os tipos de vegetação que ocorrem na Amazônia. Simpósio sobre a Biota Amazônica de 1966. Pub. Avulsas, n.20, MPEG.

Renvoize, S.A. 1984. The grasses of Babia. London: Kew publications, $301 \mathrm{p}$.

Rodrigues-Da-Silva, R.; Filgueiras, T.S. 2003. Gramíneas (Poaceae) da Área de Relevante Interesse Ecológico (ARIE) "Santuário de Vida Silvestre do Riacho Fundo", Distrito Federal, Brasil. Acta Bot. Bras., 17(3): 467-486.

Sánchez-Ken, J.G.; Clark, L.G.; Kellogg, E.A.; Kay, E.E. 2007. Reinstatement and emendation of subfamily Micrairoideae (Poaceae). Systematic Botanic, 32: 71-80.

Worbes, M. 1997. The forest ecosystem of the floodplains. In The central Amazon floodplain (W.J. Junk, ed.). Springer-Verlag, Berlin, 223-260p.

Recebido em 22/11/2007

Aceito em 24/08/2009 